\title{
The Rationale and Evidence for SGLT2 Inhibitors as a Treatment for Nondiabetic Glomerular Disease
}

\author{
Kana N. Miyata ${ }^{\text {a, }}$ Shao-Ling Zhang ${ }^{\text {a }} \quad$ John S.D. Chan ${ }^{a}$ \\ aDépartement de Médecine, Centre de Recherche du Centre Hospitalier de l'Université de Montréal (CRCHUM), \\ Université de Montréal, Montréal, QC, Canada; 'bivision of Nephrology, Department of Internal Medicine, \\ Saint Louis University School of Medicine, Saint Louis, MO, USA
}

\section{Keywords \\ Chronic kidney disease - Glomerular disease . \\ Sodium-glucose cotransporter 2 inhibitor}

\begin{abstract}
Background: Recent studies show that sodium-glucose cotransporter 2 inhibitors (SGLT2i), originally approved for glycemic control in patients with type 2 diabetes, also exert renoprotective effects independently from effects on dysglycemia. Moreover, recent work indicates that SGLT2i treatment may be effective in patients with nondiabetic chronic kidney disease, including primary and secondary glomerular diseases. Summary: SGLT2i lower blood glucose by blocking glucose resorption in the early renal proximal tubule through the glucose transporter, SGLT2, leading to enhanced urinary glucose excretion. Recent studies indicate that SGLT2i may have pleiotropic effects on cells other than proximal tubular cells. SGLT2i reduce the glomerular workload by decreasing the intraglomerular pressure, thus ameliorating hyperfiltration, if present, and may also decrease systemic blood pressure. SGLT2i may also act directly on endothelial cells, possibly via modulating the effects of adhesion molecules and reducing inflammatory cytokines and reactive oxygen species. SGLT2i may have direct anti-inflammatory and antifi-
\end{abstract}

karger@karger.com www.karger.com/gdz

Karger ${ }^{\prime \prime} \div$

BOPEN ACCESS
(C) 2021 The Author(s)

Published by S. Karger AG, Basel

This article is licensed under the Creative Commons AttributionNonCommercial-NoDerivatives 4.0 International License (CC BYNC-ND) (http://www.karger.com/Services/OpenAccessLicense) Usage and distribution for commercial purposes as well as any distribution of modified material requires written permission. brotic effects on renal tubules. Some reports suggest direct protective effects on podocytes and mesangial cells as well. Here, we provide a review of the potential mechanisms of renoprotection, therapeutic utility, and potential side effects of SGLT2i in patients with nondiabetic glomerular diseases, based on data from studies carried out in cells, experimental animals, and humans. Key Messages: SGLT2i may be a promising addition to the glomerular disease treatment armamentarium. However, it is unclear at what point of the natural history of specific glomerular diseases (whether this is immune or nonimmune mediated) SGLT2i can be beneficial. Additionally, further studies are needed to assess the longterm efficacy and safety of SGLT2i in patients with nondiabetic glomerular diseases.

(c) 2021 The Author(s)

Published by S. Karger AG, Basel

\section{Introduction}

Despite recent progress in pharmacological interventions, acute and chronic glomerular diseases continue to result in substantial morbidity and mortality [1]. The underlying etiology of glomerular diseases is diverse, resulting in many different potential therapeutic targets (Fig. 1). Currently, renin-angiotensin system (RAS) blockers are 
Fig. 1. Potential renoprotective mechanisms of SGLT2i in nondiabetic glomerular disease. Solid lines indicate SGLT2i's direct effects, and dotted lines indicate indirect effects. SGLT2i, sodium-glucose cotransporter 2 inhibitor; GEC, glomerular endothelial cell; GBM, glomerular basement membrane.

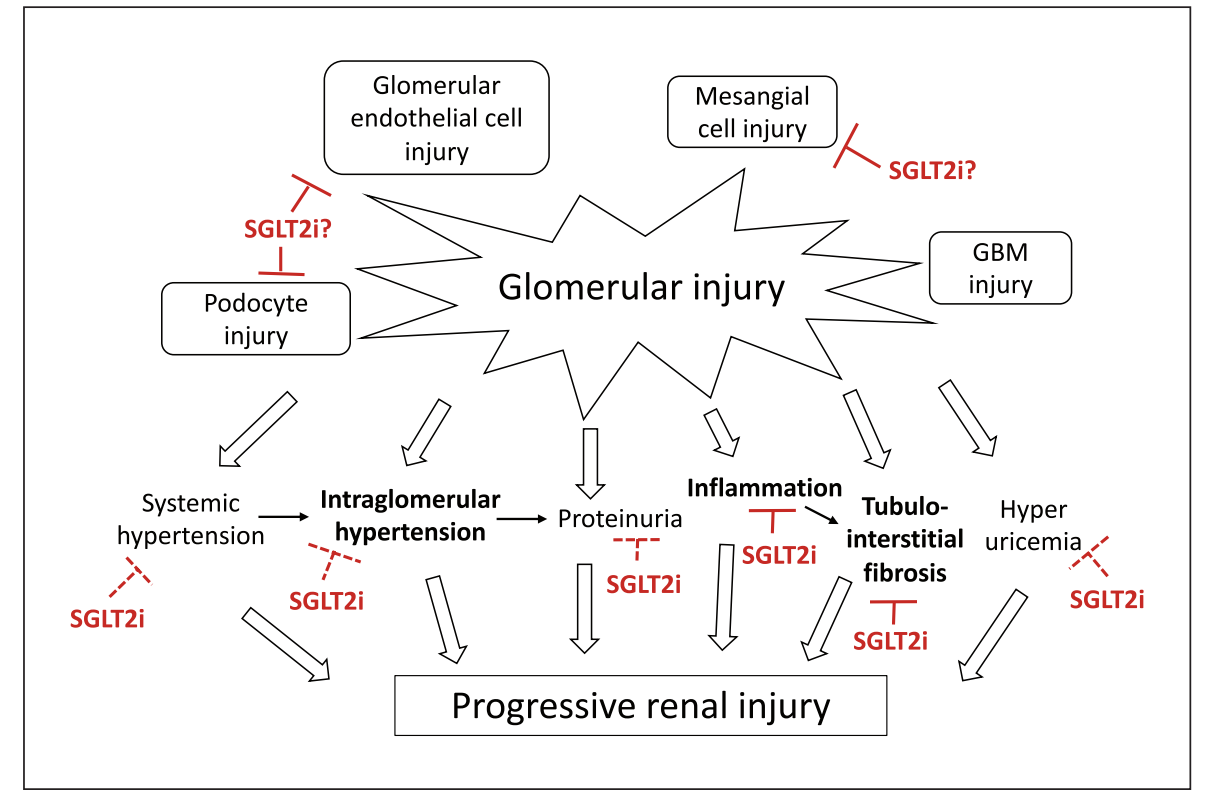

the standard of care and provide therapeutic benefits by reducing systemic and intraglomerular pressures as well as through direct effects on distinct renal cell types, acting at the molecular level.

Sodium-glucose cotransporter 2 inhibitors (SGLT2i), recently introduced as therapy for diabetes mellitus, lower blood glucose by blocking glucose entry into the renal proximal tubule cells through the SGLT2 transporter, leading to enhanced urinary glucose excretion. Recently, a number of studies in patients with type 2 diabetes (T2D) have demonstrated that SGLT2i unexpectedly exerted renoprotective effects independently of its glucose-lowering effects [2-4]. Moreover, it appears that SGLT2i may also have pleiotropic effects at the cellular level beyond the proximal tubule. Further, a recent randomized controlled trial (RCT), DAPA-CKD, has demonstrated that SGLT2i slowed the progression of diabetic and nondiabetic CKD [5]. Taken together, data suggest that SGLT2i may have a place in standard therapy of CKD.

Glomerular diseases can be immune- or nonimmunemediated damages. From the current evidence, it appears that SGLT2i would not target the pathomechanistic etiologies of immune-mediated glomerular diseases. Rather, it may ameliorate the downstream injury from the original glomerular damages (Fig. 1).

Why do SGLT2i, which primarily act in the proximal tubules, have upstream effects on glomeruli and elsewhere? In this review, we consider various studies from cells and animal models to humans and summarize the proposed mechanisms through which SGLT2i may provide renoprotection in nondiabetic glomerular diseases (Fig. 1). Since studies of SGLT2i employed specifically for nondiabetic CKD both in humans and animals are limited (Tables 1,2) [5-16], we highlight the implications of relevant results from studies in diabetes as well. Finally, we consider whether SGLT2i constitute a potential treatment option for patients with nondiabetic glomerular diseases.

\section{SGLTs and Their Effects}

Under euglycemic condition, the kidney reabsorbs almost all the glucose filtered in the glomerulus through two glucose transporters: sodium-glucose cotransporter 1 (SGLT1) and SGLT2. SGLT2 is a low-affinity, high-capacity glucose transporter, expressed in the apical membrane of the early S1/S2 segments of the proximal tubules, which accounts for $>90 \%$ of the glucose reabsorption [17]. SLC5A2 encodes SGLT2 and is a causative gene for familial renal glycosuria. The remaining luminal glucose reaches the downstream S2/S3 segments of the proximal tubule and is reabsorbed by the high-affinity, low-capacity glucose transporter SGLT1. SGLT1 is encoded by SLC5A1 and is also expressed in the mucosa of the small intestine [18]. Glucose reabsorption through SGLT1 has been reported to increase when SGLT2 is not expressed (SLC5A2 gene is deleted) or is inhibited by SGLT2i or when filtered glucose is increased in the setting of diabetes [17]. 


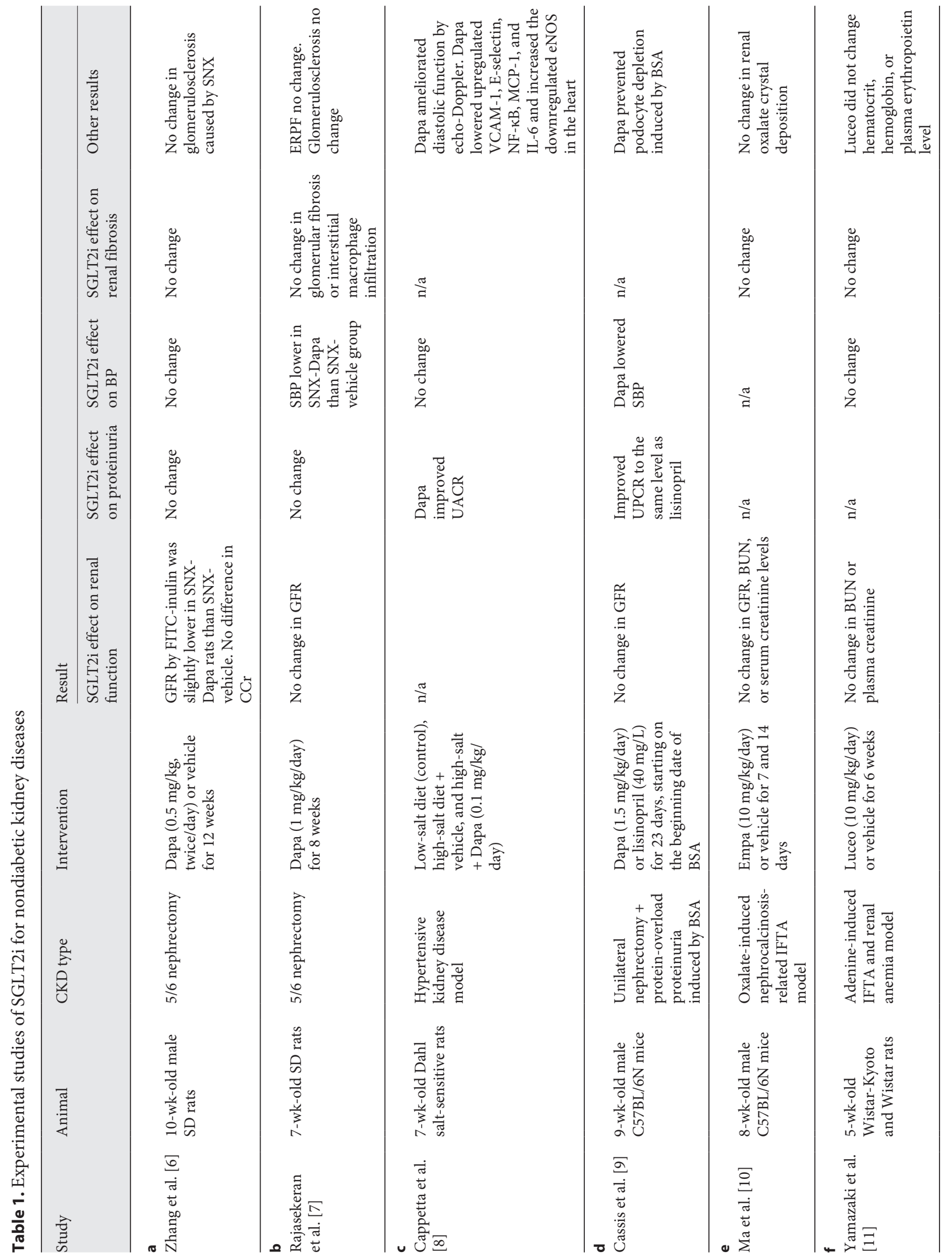




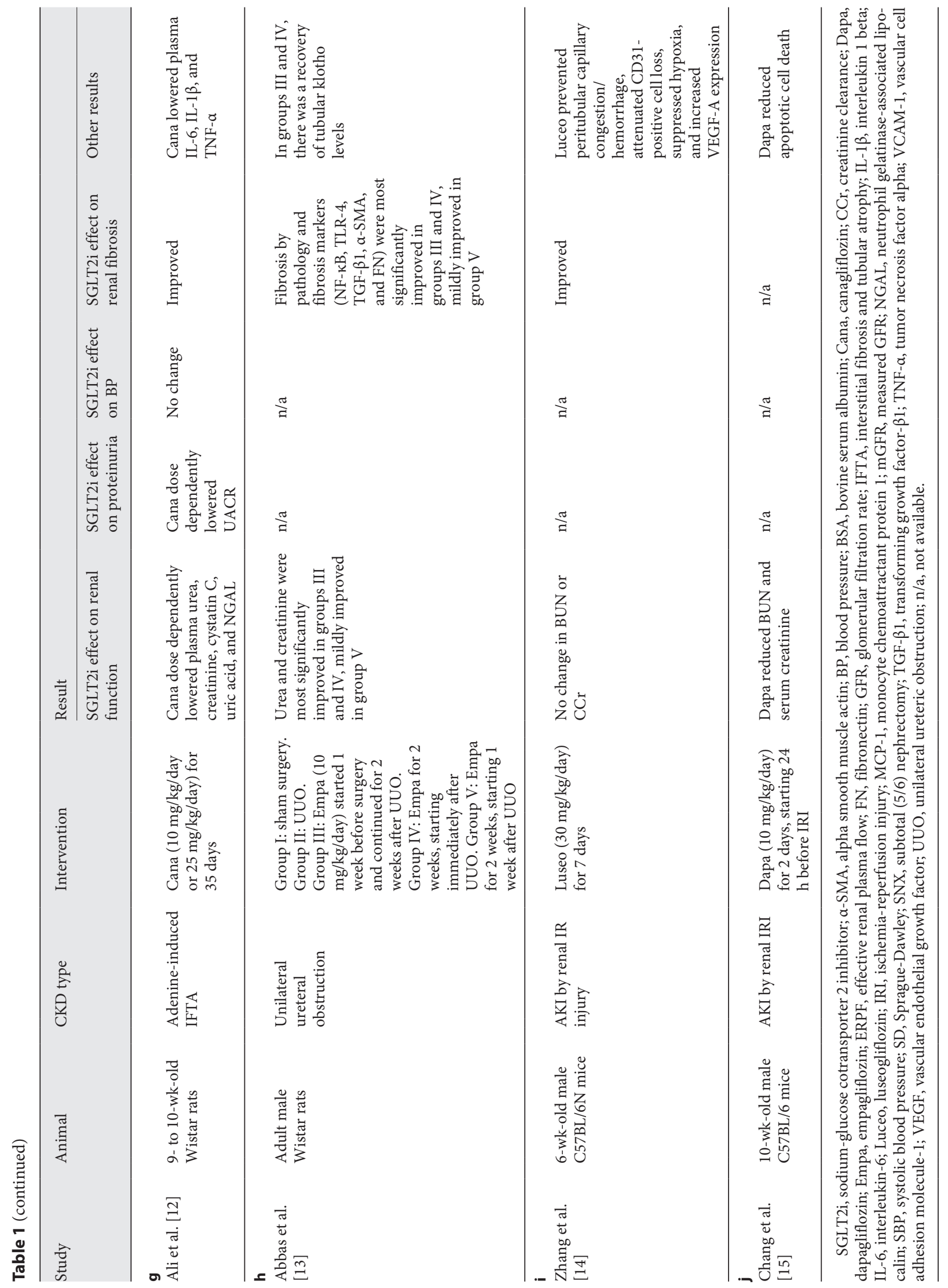




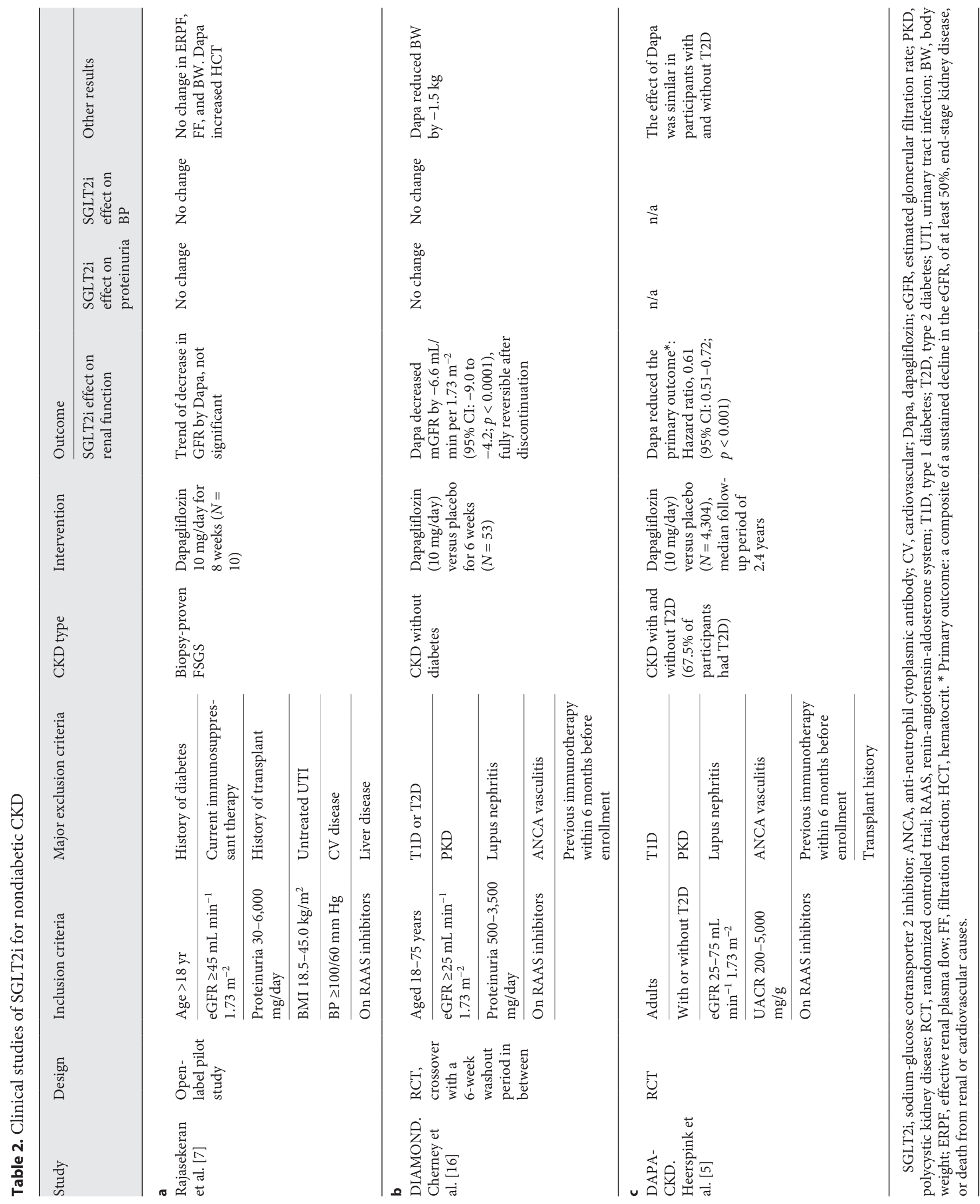




\section{Tubular SGLT2 Expression in Glomerular Diseases}

An important point to discuss when considering the expansion of SGLT2i treatment in nondiabetic glomerular diseases is the difference of SGLT2 expression between diabetic and nondiabetic kidneys. SGLT2 expression abundance has been most frequently studied in patients and animal models with diabetes compared to healthy controls without diabetes. Most animal studies have shown increased SGLT2 expression in both type 1 diabetes (T1D) and T2D, except for streptozotocin-induced diabetic mice $[19,20]$. In humans, there are conflicting results in SGLT2 abundance in diabetic patients versus nondiabetic healthy controls $[21,22]$. Therefore, a concern has been raised whether the use or effect of SGLT2i in nondiabetic patients is comparable to that in patients with diabetes. It is also not known whether the efficacy of SGLT2i corresponds to the level of SGLT2 expression. Indeed, Rajasekeran et al. [7] reported that renal SGLT2 mRNA levels in kidney biopsies from patients with obesity-related secondary FSGS $(n=6)$ were significantly lower than the levels in healthy controls (transplant donor kidney, $n=6$ ). In contrast, data from the Nephrotic Syndrome Study Network (NEPTUNE) demonstrated that tubular SGLT2 mRNA levels were the same in biopsies from patients with glomerulonephritis and kidney tissue from normal controls (kidney donors), and the expressions were similar across different subtypes of nephrotic syndrome (membranous nephropathy, minimal change disease, FSGS, and IgA nephropathy) [22]. Tubular SGLT2 mRNA expression was positively correlated with estimated GFR (eGFR) and glycated hemoglobin and inversely correlated with interstitial fibrosis, suggesting that CKD stages may be the key factor affecting the SGLT2 expression [22]. Furthermore, recent evidence from the DAPA-HF and DAPA-CKD trials supports the concept that SGLT2i may function even in nondiabetic states, as both cardio- and renoprotective benefits of dapagliflozin were similar in patients with or without diabetes $[5,23]$.

\section{Effects of SGLT2i on Intraglomerular Pressure}

Nephron loss from glomerular disease leads to a compensatory response in spared nephrons, with increased intraglomerular pressure, glomerular hypertrophy, and hyperfiltration, leading to further glomerular damage in those nephrons that remain [24]. Reduction of the glomerular workload through decreasing the intraglomeru- lar pressure has long been considered a viable strategy to preserve the remaining nephron function for many forms of CKD. The management of CKD based on this concept includes low-protein diet and RAS blockade, but recent studies have revealed that SGLT2i also affect the intraglomerular hemodynamics, with resultant decrease in intraglomerular pressure.

The mechanisms of improved renal hemodynamics with SGLT2i are incompletely understood. Both afferent and efferent renal arteriolar pathway theories have been proposed via preglomerular vasoconstriction induced by the activation of tubulo-glomerular feedback (TGF) [25] and postglomerular vasodilation [26], respectively. TGF is a complex autoregulatory feedback system that controls the GFR based on the amount of $\mathrm{NaCl}$ delivered to the macula densa at the juxtaglomerular apparatus [27]. When the macula densa senses increased $\mathrm{NaCl}$, it promotes the adenosine release. Adenosine, via A1 receptor activation on the afferent arteriole, activates phospholipase $\mathrm{C}$, followed by inositol triphosphate and diacylglycerol release, which results in cellular depolarization and influx of intracellular calcium, leading to afferent arteriole constriction and a fall in GFR. SGLT2 inhibition, by blocking the sodium reabsorption at the proximal tubule, increases the distal sodium delivery and therefore decreases the GFR via TGF. An additional postulated mechanism of increased sodium delivery to the macula densa is that SGLT2i may also decrease the activity of $\mathrm{Na}^{+} / \mathrm{H}^{+}$ exchanger 3 (NHE3), another sodium transporter colocalized with the SGLT2, thereby blocking much of the sodium reabsorption at the proximal tubules [28]. The mechanism of the functional link between NHE3 and SGLT2 is not clearly understood, but it has been proposed that $17-\mathrm{kDa}$ membrane-associated protein/NHE-regulatory cofactor 3 and/or NHE3 phosphorylation might be involved [29].

We and others have reported that SGLT2i decrease the GFR in T1D (Akita) and T2D ( $d b / d b)$ mouse models [3032]. Moreover, by using in vivo multiphoton microscopy techniques, Kidokoro et al. [33] showed that increased single-nephron GFR in Akita mice was decreased by empagliflozin treatment, and that an A1 adenosine receptor antagonist, which blocks the TGF, attenuated the empagliflozin's effect on the GFR. This observation suggests that adenosine signaling in the TGF may be a key regulator of the glomerular hemodynamic change with SGLT2i. The effect of the SGLT2i on GFR was also determined in T1D patients: empagliflozin treatment for 8 weeks significantly reduced the measured GFR (inulin clearance) in patients with hyperfiltration at baseline $(N=27)$ [25]. 
This hemodynamic change is thought to account for the initial acute drop of eGFR reported in patients with T2D, despite subsequent stabilization of the eGFR [2-4].

An important question is whether the reduction of intraglomerular pressure by SGLT2i can also be achieved in nondiabetic CKD. Using 5/6 nephrectomized rats, a model of progressive CKD that resembles FSGS in humans, Zhang et al. [6] reported that reduced GFR (measured by FITC-inulin clearance) induced by $5 / 6$ nephrectomy was slightly further decreased by dapagliflozin treatment for 12 weeks (Table 1a). However, these authors also showed that dapagliflozin did not improve proteinuria or glomerular injury by histopathological examination [6]. In contrast, Rajasekeran et al. [7] reported that dapagliflozin treatment for 8 weeks did not change GFR or proteinuria in rats with subtotal nephrectomy (Table 1b). In clinical studies, in a small pilot study of dapagliflozin treatment for 8 weeks in FSGS patients $(n=$ 10 ), there was a nonsignificant trend of decrease in GFR and effective renal plasma flow in the dapagliflozin group (Table 2a) [7]. Recently, the same group published the DIAMOND trial, the first randomized double-blind clinical study of SGLT2i on patients with nondiabetic CKD who were on stable RAS blockers at baseline (Table $2 b$, $n=53$, including patients with IgA nephropathy, FSGS, hypertensive nephropathy, and other pathologies), which showed a significant reduction of measured GFR (via iohexol clearance) by $-6.6 \mathrm{~mL} / \mathrm{min}$ per $1.73 \mathrm{~m}^{2}$ (-9.0 to $-4.2 ; p<0.0001)$ after 6 weeks of treatment with dapagliflozin that was fully reversible after discontinuation of the drug, though proteinuria was unchanged during the observation [16]. These results suggest that SGLT2i likely exert renal hemodynamic functional changes in humans who do not have diabetes. Clearly, longer-term studies are warranted to confirm whether this renal hemodynamic change will lead to the overall beneficial effect on renal outcome in patients with nondiabetic glomerular diseases.

\section{Effects of SGLT2i on Systemic BP}

Since the afferent arteriole transmits systemic blood pressure (BP) to each glomerulus, affecting the intraglomerular pressure and protein leak, systemic BP normalization in patients with hypertension is one of the most important CKD management strategies. It has been accepted that decrease in BP is part of the unique nonglycemic effects of SGLT2i. Meta-analysis of 43 RCTs with 22,528 T2D patients revealed that SGLT2i consistently induced a reduction of $\mathrm{BP}$, mean difference of $-2.46 \mathrm{~mm}$ $\mathrm{Hg}$ (95\% confidence interval [CI]: -2.86 to -2.06 ) for systolic and $-1.46 \mathrm{~mm} \mathrm{Hg}(95 \% \mathrm{CI}:-1.82$ to -1.09$)$ for diastolic BP [34]. Moreover, RCTs of patients with T1D have shown that SGLT2i lower BP dose dependently $[35,36]$.

Such reports have results counter to published animal studies, including our reports, in which reduction of BP is inconsistent [30,31,37]. One intriguing report showed that luseogliflozin did not reduce $\mathrm{BP}$ in T2D rats, but the combination of luseogliflozin and lisinopril significantly reduced $\mathrm{BP}$ more than that seen in the rats treated with lisinopril monotherapy, suggesting that SGLT2i and RAS blockers may act in concert to lower the systemic BP [38]. Another report demonstrated that systolic BP was lowered by dapagliflozin in 5/6 nephrectomized rats (Table 1b) [7]. In contrast, in Dahl salt-sensitive rats, dapagliflozin did not reduce the BP induced by high-salt diet (Table 1c) [8]. In other nondiabetic studies, both in animals and patients, in which SGLT2i were used, significant changes of BP were not observed (Tables 1, 2). Clearly, further studies are warranted to assess whether the BP lowering effects of SGLT2i in T2D occur in nondiabetic glomerular diseases.

The mechanisms of BP lowering with SGLT2i appear complex and multifactorial. Possible contributing factors include decreased plasma volume, weight loss, better glycemic control (in patients with diabetes or prediabetes), attenuation of inflammation, and improved arterial stiffness and endothelial function $[39,40]$. Among these factors, the most important is likely the diuretic effects of SGLT2i. The chronic osmotic diuresis and natriuretic effects of SGLT2i cause plasma volume contraction, leading to reduced arterial pressure. This likely mechanism is supported by the observed increase in hematocrit and reductions in body weight, both of which are also consistently reported in clinical trials with both T1D $[35,36]$ and T2D $[3,4]$ patients. Of note, the increased hematocrit may, in part, be due to the enhancement of erythropoietin production by SGLT2i [39].

Similarly, the effect of SGLT2i on the RAS has yielded discordant results. In a clinical study in which empagliflozin was given for 8 weeks in T1D patients $(N=40)$, empagliflozin increased both systemic and intrarenal RAS, without affecting the plasma renin activity $[25,41]$. In contrast, others reported no change in urinary angiotensinogen (Agt) after 1-month treatment with 5 different SGLT2i in T2D patients $(N=9)$ [42]. Our group has performed preclinical studies to investigate whether SGLT2i modulate the intrarenal RAS. We showed that renal Agt expression was similar between nondiabetic wild type and wild 
type treated with canagliflozin for 4 weeks [43]. Moreover, renal Agt mRNA, protein expression, and urinary Agt excretion were unchanged in Akita mice treated with canagliflozin compared to Akita mice, though blood glucose, GFR, glomerular injury, and renal fibrosis were all significantly ameliorated by canagliflozin [31]. However, a study by Woods et al. [37] showed that canagliflozin given to T2D mice ameliorated hyperglycemia-associated augmentation of renal AGT expression. Additional studies are needed to clarify whether these conflicting results are related to differences between T1D and T2D and whether the intrarenal/systemic RAS could be altered by SGLT2i under both euglycemic and hyperglycemic conditions.

\section{Effect of SGLT2i on Endothelial Cells}

The glomerular filtration barrier consists of glomerular endothelial cells (GECs), the tripartite glomerular basement membrane, and podocytes. GEC injury is seen in both primary glomerular diseases and in systemic conditions such as diabetes, lupus nephritis, thrombotic microangiopathy, and vasculitis. Interestingly, there is accumulating evidence suggesting that SGLT2i may also directly act on the endothelial cells.

To the best of our knowledge, there is no report of SGLT2i specifically inducing changes in GECs. Because glucose-independent cardiovascular benefits have been consistently observed in clinical studies with SGLT2i, multiple animal, ex vivo and in vitro studies have been performed to explore their effects on cardiovascular endothelial cells under diabetic and nondiabetic conditions [44]. As an example of euglycemic animal studies, dapagliflozin decreased the overexpression of VCAM-1 and E-selectin and restored the downregulated eNOS levels in the hearts of Dahl salt-sensitive rats, a model of hypertension (Table 1c) [8]. In the same study, the authors also showed upregulation of endothelial $\mathrm{Na}^{+} / \mathrm{H}^{+}$exchanger 1 (NHE1) levels in vivo and direct effects of dapagliflozin on the activity of NHE1 in human umbilical vein endothelial cells (HUVECs) in vitro [8]. Furthermore, in healthy rats and rabbits, an endothelium-dependent vasorelaxation was induced by canagliflozin and dapagliflozin [44]. In the atherosclerosis model of ApoE knockout mice, empagliflozin for 8 weeks lowered circulating levels of tumor necrosis factor alpha (TNFa), interleukin-6 (IL-6), monocyte chemoattractant protein 1 (MCP-1), and high-sensitivity C-reactive protein [45].

In cell culture studies, canagliflozin prevented IL-1 $\beta$ induced IL- 6 and MCP-1 expression in HUVECs and hu- man aortic endothelial cells [46]. Dapagliflozin also prevented TNFa-induced increases of ICAM-1 and VCAM1 protein in HUVECs [47]. Moreover, empagliflozin and dapagliflozin attenuated TNFa-induced reactive oxygen species (ROS) generation in human coronary artery endothelial cells and HUVECs [48]. These consistent experimental studies implicate a possible class effect of SGLT2i on reducing inflammation in endothelial cells, leading to improved endothelial function.

Whether SGLT2i are actually targeting SGLT2 in endothelial cells or these actions are mediated via other transporters such as NHE1 are currently under investigation. Evidence indicates that there is both SGLT2 mRNA and protein expression in endothelial cells of mouse aortic tissue and HUVECs $[44,49]$. However, there is also a report of positive SGLT2 protein in Western blotting without detectable mRNA in human coronary artery endothelial cells [48]. Recently, Khemais-Benkhiat et al. [50] reported that SGLT2 mRNA was not detectable under normal conditions, but became above the detection level after $\mathrm{H}_{2} \mathrm{O}_{2}$ or high glucose exposure. These results suggest that the baseline SGLT2 expression in normal endothelial cells may be small, but it may be enhanced in various disease states. Although the presence of SGLT2 in endothelial cells may remain controversial, there is increasing evidence suggesting that SGLT2i likely act directly on endothelial cells, possibly via modulating adhesion molecules and reducing inflammatory cytokines and/or ROS.

\section{Effect of SGLT2i on Mesangial Cells}

Though SGLT2 is primarily expressed in the renal proximal tubules, SGLT2 expression has been reported to be present in mouse mesangial cells [51]. Low-dose administration of canagliflozin, which did not lower the blood glucose level, improved albuminuria and mesangial expansion in type $2 d b / d b$ mice [51]. In cultured mouse mesangial cells, high glucose exposure increased SGLT2 expression, and canagliflozin treatment inhibited high glucose-induced protein kinase $\mathrm{C}$ activation and ROS production [51]. This is also supported by one of our previous reports, in which the mesangial expansion in T1D Akita mice was more significantly improved by canagliflozin than by insulin, though both canagliflozintreated and insulin-treated groups maintained similar blood glucose levels for 4 weeks [31]. Further studies are warranted to investigate SGLT2 expression and the direct effects of SGLT2i on mesangial cells.
28

Glomerular Dis 2021;1:21-33

DOI: $10.1159 / 000513659$
Miyata/Zhang/Chan 


\section{Effect of SGLT2i on Podocytes}

Cassis et al. [9] reported in studies using immunofluorescence and Western blotting techniques that SGLT2 is expressed in mouse and human podocytes. Using mice with protein-overload proteinuria induced by bovine serum albumin, Cassis et al. [9] showed that dapagliflozin ameliorated the proteinuria, glomerular lesions, and foot process effacement (Table 1d). In vitro, SGLT2 expression by Western blotting was stimulated by albumin exposure in cultured human podocytes, and dapagliflozin ameliorated albumin-induced cytoskeletal rearrangements in podocytes [9]. Compared to normal human kidney tissue in tumor nephrectomy specimens, biopsy samples from 4 patients with idiopathic membranous nephropathy had increased SGLT2 expression in podocytes by immunofluorescence staining. Conclusions are tentative, given the pilot nature of that work [9].

\section{Effect of SGLT2i on Tubulointerstitial Fibrosis}

Regardless of the etiology, the most common pathological manifestation at the final stage of CKD is renal fibrosis. Renal fibrosis may be considered as a failed wound healing process following various insults to the kidneys. Even if the primary cause of CKD is glomerular injury, the best predictor of renal functional decline is actually the extent of interstitial fibrosis [52]. Key mediators of fibrosis are inflammatory cell infiltration, myofibroblast activation and excessive extracellular matrix production, and, ultimately, tubular cell atrophy and epithelial-mesenchymal transition (EMT) [52]. Interestingly, multiple recent studies have revealed that SGLT2i may have antifibrotic effects by interfering with the profibrotic steps just outlined.

Many diabetic animal studies have shown decrease in interstitial fibrosis after treatment with SGLT2i $[32,37$, 38]. In such models of diabetes, however, it is difficult to differentiate direct antifibrotic effects from secondary effects caused by the improvement in dysglycemia and hemodynamic changes. Thus, it is important to assess the effects of SGLT2i against active controls. In our study with T1D Akita mice treated for 4 weeks to similar glucose levels with either canagliflozin or insulin, we observed similar improvement of renal fibrosis [31]. Li et al. [53] studied streptozotocin-induced T1D mice treated with empagliflozin or insulin to adjust the glucose level for a month and showed that empagliflozin but not insulin suppressed kidney fibrosis. Moreover, in the study by

SGLT2 Inhibitors for Nondiabetic

Glomerular Disease
Li et al. [53], empagliflozin but not insulin suppressed the EMT in the renal proximal tubules.

One clinical study addressed the direct antifibrotic effect of SGLT2i by comparing patients who were treated either with canagliflozin or glimepiride to a similar glycemic level $(N=296)$ [54]; canagliflozin $300 \mathrm{mg} /$ day decreased plasma levels of TNF receptor 1 (TNFR1), IL-6, matrix metalloproteinase 7 , and fibronectin 1 (FN1) more significantly than glimepiride during the 2-year followup.

Some compelling evidence supports direct antifibrotic effects of SGLT2i in vitro. Empagliflozin attenuated high glucose-induced toll-like receptor-4 (TLR-4) expression and increased nuclear deoxyribonucleic acid binding for nuclear factor kappa B (NF-kB), IL-6 secretion, and collagen IV expression in human immortalized proximal tubular cells (HK2) [55]. Dapagliflozin treatment also reduced high glucose-induced overexpression of alpha smooth muscle actin ( $\alpha$-SMA) and increased STAT1 and transforming growth factor- $\beta 1$ (TGF- $\beta 1$ ) expression in HK2 cells [56]. Furthermore, SGLT2 knockdown in HK2 cells resulted in protection from high glucose-induced EMT [53].

In contrast, in animal models of nondiabetic renal fibrosis, the beneficial effect of SGLT2i has not been shown consistently. In mice with tubular damage induced by chronic oxalosis without direct glomerular damage, empagliflozin did not improve renal function or fibrosis (Table 1e) [10]. In the adenine-induced interstitial fibrosis and tubular atrophy (IFTA) model in rats, luseogliflozin for 6 weeks did not improve renal function or fibrosis (Table 1f) [11], but canagliflozin for 35 days dose dependently improved renal function and urinary albumin excretion as well as IFTA (Table 1g) [12]. In a model of unilateral ureteric obstruction (UUO) in rats, 2-week treatment with empagliflozin improved renal function and renal fibrosis, accompanied by reduced inflammatory and fibrotic markers in the kidney, such as NF-kB, TLR-4, TGF- $\beta 1, \alpha-S M A$, and FN (Table 1h) [13]. In this study of the UUO model [13], the most significant improvement of renal fibrosis was observed in groups which were prophylactically treated with empagliflozin before UUO and those treated immediately after UUO. Intriguingly, the third treatment group of mice which was started on empagliflozin 1 week after UUO (delayed treatment) also had mild but significant improvement of renal function and fibrosis [13]. These observations are promising for the translation to the clinical setting because most of the CKD patients have some degree of fibrosis at the time of diagnosis and pharmacological intervention. 
Taken together, there appears to be direct anti-inflammatory and antifibrotic effects conferred by SGLT2i. However, considering the discordant nondiabetic animal study results in different fibrosis models, further studies are needed to establish the evidence that SGLT2i can improve renal fibrosis in nondiabetic glomerular diseases.

\section{Effect of SGLT2i on Renal Uric Acid Handling}

Clinical trials have shown that SGLT2i treatment leads to a modest but significant reduction in the serum uric acid concentration through increased uricosuria [57]. Although the mechanism is not clearly understood, studies suggest that SGLT2 inhibition changes the activity of GLUT-9 isoform b, reducing urate reabsorption [58]. Hyperuricemia is a common finding in CKD patients, and it has been linked to tubulointerstitial fibrosis progression through the induction of inflammation, oxidative stress, endothelial dysfunction, and RAS activation. Therefore, SGLT2 inhibition may possibly indirectly contribute to renal tubular protection by lowering serum uric acid levels.

\section{Effects of SGLT2i on Renal Function in Clinical Studies in Patients with Nondiabetic CKD}

Several recently published clinical trials have included patients with nondiabetic CKD (Table 2) or are currently in progress to investigate the renal benefits of SGLT2i $[5,7,16,59]$. In the DAPA-CKD study (Table 2c), 4,304 patients with and without T2D were randomized to receive dapagliflozin $10 \mathrm{mg}$ /day or placebo. The trial was stopped early because of the "overwhelming efficacy" of dapagliflozin. Over a median follow-up period of 2.4 years, the primary endpoint (a composite of sustained decline in the eGFR of at least $50 \%$, end-stage kidney disease, or death from renal or cardiovascular causes) was significantly attenuated by dapagliflozin (hazard ratio, 0.61; 95\% CI: $0.51-0.72$; $p<0.001)$. The effects of dapagliflozin were similar in participants with $\mathrm{T} 2 \mathrm{D}(67.5 \%$ of participants) and without T2D (etiologies of CKD in those without T2D were reported as ischemic/hypertensive nephropathy [16\%], IgA nephropathy [6.3\%], FSGS [2.7\%], membranous nephropathy [1\%], minimal change disease $[0.3 \%]$, chronic pyelonephritis [1.6\%], chronic interstitial nephritis [1.2\%], obstructive nephropathy [0.6\%], and others) [5]. Both in DIAMOND and DAPACKD studies, patients with lupus nephritis, polycystic kidney disease, and vasculitis were excluded. Another large-scale long-term RCT with empagliflozin, EMPAKidney (NCT03594110), is currently ongoing and expected to be completed in 2022 [59].

Currently, it is undetermined until which level of reduced kidney function the SGLT2i exert renoprotective function. Although some studies have shown that SGLT2i's glucose-lowering effect becomes less in patients with lower GFR, it has been suggested that improvement of BP and proteinuria by SGLT2i may persist in advanced CKD $[60,61]$.

\section{Potential Side Effects of SGLT2i}

Genital mycotic infections are the most common side effects as described in studies of SGLT2i in T2D patients, but the majority of the infectious complications are mild to moderate which respond to topical antifungals [62]. Patients with glomerular diseases also tend to be on immunosuppressive medications and are susceptible to infections. Therefore, patient education and self-monitoring are important.

Because of the osmotic and natriuretic diuresis properties, SGLT2i may predispose patients to dehydration, hypotension, and potentially acute kidney injury. In the DAPA-CKD study, volume depletion was slightly more frequent in the dapagliflozin group ( 5.9 vs. $4.2 \% ; p=0.01$ ) [5]. It may need a closer monitoring especially for those who are on other diuretic drugs. Canagliflozin was initially found to be associated with a higher risk of lowerlimb amputation and fractures in the CANVAS trial, but later trials such as CREDENCE, DAPA-HF, and DAPACKD showed similar rates, and meta-analysis has not proven the associations [4].

\section{Conclusions}

Multiple clinical trials with SGLT2i in patients with diabetes have raised the possibility that this class of drugs may provide renoprotection independent of its antiglycemic effects. Accumulating evidence suggests that SGLT2i may act on many different types of kidney cells and in distinct kidney compartments with specific and varied molecular and cellular effects. Given the recent renoprotective effects observed in the DAPA-CKD trial [5], the use of SGLT2i in CKD unrelated to diabetes appears promising. However, it is unclear at what point of the natural history of specific glomerular diseases (whether this is im-
30

Glomerular Dis 2021;1:21-33 DOI: $10.1159 / 000513659$
Miyata/Zhang/Chan 
mune or nonimmune mediated) SGLT2i can be beneficial. Therefore, more studies targeting nondiabetic primary and secondary glomerular diseases are warranted to assess the efficacy and safety of SGLT2i in patients with glomerular diseases that are not associated with diabetes.

\section{Acknowledgement}

We thank Julie R. Ingelfinger, MD, for advice and editing suggestions relevant to this manuscript.

\section{Conflict of Interest Statement}

All the authors declare that there are no conflicts of interest relevant to this article.

\section{Funding Sources}

This work was supported, in part, by grants from the Canadian Institutes of Health Research (MOP-84363 and MOP-142378 to J.S.D.C. and MOP-86450 to S.L.Z.) and the Kidney Foundation of Canada (KFOC 170006 to J.S.D.C.). K.N.M. is a recipient of a fellowship from the Consortium de Néphrologie de l'Université de Montréal (2018) and Ben J. Lipps Research Fellowship Program of the American Society of Nephrology (2019-2020).

\section{Author Contributions}

K.N.M. performed the literature search and drafted the article. S.L.Z. and J.S.D.C. reviewed/edited the manuscript. All authors approved the final version for publication.

\section{References}

1 Webster AC, Nagler EV, Morton RL, Masson P. Chronic kidney disease. Lancet. 2017; 389(10075):1238-52.

2 Wanner C, Inzucchi SE, Lachin JM, Fitchett D, von Eynatten M, Mattheus M, et al. Empagliflozin and progression of kidney disease in type 2 diabetes. N Engl J Med. 2016;375(4): 323-34.

3 Perkovic V, Jardine MJ, Neal B, Bompoint S, Heerspink HJL, Charytan DM, et al. Canagliflozin and renal outcomes in type 2 diabetes and nephropathy. N Engl J Med. 2019; 380(24):2295-306.

4 Neal B, Perkovic V, Mahaffey KW, de Zeeuw D, Fulcher G, Erondu N, et al. Canagliflozin and cardiovascular and renal events in type 2 diabetes. N Engl J Med. 2017;377(7):644-57.

5 Heerspink HJL, Stefánsson BV, Correa-Rotter R, Chertow GM, Greene T, Hou FF, et al. Dapagliflozin in patients with chronic kidney disease. N Engl J Med. 2020;383(15): 1436-46.

6 Zhang Y, Thai K, Kepecs DM, Gilbert RE. Sodium-glucose linked cotransporter-2 inhibition does not attenuate disease progression in the rat remnant kidney model of chronic kidney disease. PLoS One. 2016; 11(1):e0144640.

7 Rajasekeran H, Reich HN, Hladunewich MA, Cattran D, Lovshin JA, Lytvyn Y, et al. Dapagliflozin in focal segmental glomerulosclerosis: a combined human-rodent pilot study. Am J Physiol Renal Physiol. 2018;314(3): F412-22.

8 Cappetta D, De Angelis A, Ciuffreda LP, Coppini R, Cozzolino A, Miccichè A, et al. Amelioration of diastolic dysfunction by dapagliflozin in a non-diabetic model involves coronary endothelium. Pharmacol Res. 2020; 157:104781.
9 Cassis P, Locatelli M, Cerullo D, Corna D, Buelli S, Zanchi C, et al. SGLT2 inhibitor dapagliflozin limits podocyte damage in proteinuric nondiabetic nephropathy. JCI Insight. 2018;3(15):e98720.

$10 \mathrm{Ma}$ Q, Steiger S, Anders HJ. Sodium glucose transporter-2 inhibition has no renoprotective effects on non-diabetic chronic kidney disease. Physiol Rep. 2017;5(7):e13228.

11 Yamazaki D, Konishi Y, Morikawa T, Kobara $\mathrm{H}$, Masaki T, Hitomi H, et al. Failure to confirm a sodium-glucose cotransporter 2 inhibitor-induced hematopoietic effect in non-diabetic rats with renal anemia. J Diabetes Investig. 2020;11(4):834-43.

12 Ali BH, Al Salam S, Al Suleimani Y, Al Za'abi $M$, Abdelrahman AM, Ashique M, et al. Effects of the SGLT-2 inhibitor canagliflozin on adenine-induced chronic kidney disease in rats. Cell Physiol Biochem. 2019;52(1):27-39.

13 Abbas NAT, El Salem A, Awad MM. Empagliflozin, SGLT2 inhibitor, attenuates renal fibrosis in rats exposed to unilateral ureteric obstruction: potential role of klotho expression. Naunyn Schmiedebergs Arch Pharmacol. 2018;391(12):1347-60.

14 Zhang Y, Nakano D, Guan Y, Hitomi H, Uemura A, Masaki T, et al. A sodium-glucose cotransporter 2 inhibitor attenuates renal capillary injury and fibrosis by a vascular endothelial growth factor-dependent pathway after renal injury in mice. Kidney Int. 2018; 94(3):524-35.

15 Chang YK, Choi H, Jeong JY, Na KR, Lee KW, Lim BJ, et al. Dapagliflozin, SGLT2 inhibitor, attenuates renal ischemia-reperfusion injury. PLoS One. 2016;11(7):e0158810.
16 Cherney DZI, Dekkers CCJ, Barbour SJ, Cattran D, Abdul Gafor AH, Greasley PJ, et al. Effects of the SGLT2 inhibitor dapagliflozin on proteinuria in non-diabetic patients with chronic kidney disease (DIAMOND): a randomised, double-blind, crossover trial. Lancet Diabetes Endocrinol. 2020;8(7):582-93.

17 Rieg T, Masuda T, Gerasimova M, Mayoux E, Platt K, Powell DR, et al. Increase in SGLT1mediated transport explains renal glucose reabsorption during genetic and pharmacological SGLT2 inhibition in euglycemia. Am J Physiol Renal Physiol. 2014;306(2):F188-93.

18 Gorboulev V, Schürmann A, Vallon V, Kipp $\mathrm{H}$, Jaschke A, Klessen D, et al. Na+-d-glucose cotransporter SGLT1 is pivotal for intestinal glucose absorption and glucose-dependent incretin secretion. Diabetes. 2012;61(1):18796.

19 Vallon V, Rose M, Gerasimova M, Satriano J, Platt KA, Koepsell H, et al. Knockout of Naglucose transporter SGLT2 attenuates hyperglycemia and glomerular hyperfiltration but not kidney growth or injury in diabetes mellitus. Am J Physiol Renal Physiol. 2013; 304(2):F156-67.

20 Umino H, Hasegawa K, Minakuchi H, Muraoka H, Kawaguchi T, Kanda T, et al. High basolateral glucose increases sodium-glucose cotransporter 2 and reduces sirtuin- 1 in renal tubules through glucose transporter-2 detection. Sci Rep. 2018;8(1):6791.

21 Wang XX, Levi J, Luo Y, Myakala K, HermanEdelstein M, Qiu L, et al. SGLT2 protein expression is increased in human diabetic nephropathy: SGLT2 protein inhibition decreases renal lipid accumulation, inflammation, and the development of nephropathy in diabetic mice. J Biol Chem. 2017;292(13):5335-48. 
22 Srinivasan Sridhar V, Ambinathan JPN, Kretzler M, Pyle LL, Bjornstad P, Eddy S, et al. Renal SGLT mRNA expression in human health and disease: a study in two cohorts. Am J Physiol Renal Physiol. 2019;317(5):F122430.

23 McMurray JJV, Solomon SD, Inzucchi SE, Køber L, Kosiborod MN, Martinez FA, et al. Dapagliflozin in patients with heart failure and reduced ejection fraction. N Engl J Med. 2019;381(21):1995-2008.

24 Helal I, Fick-Brosnahan GM, Reed-Gitomer B, Schrier RW. Glomerular hyperfiltration: definitions, mechanisms and clinical implications. Nat Rev Nephrol. 2012;8(5):293-300.

25 Cherney DZ, Perkins BA, Soleymanlou N, Maione M, Lai V, Lee A, et al. Renal hemodynamic effect of sodium-glucose cotransporter 2 inhibition in patients with type 1 diabetes mellitus. Circulation. 2014;129(5):587-97.

26 van Bommel EJM, Muskiet MHA, van Baar MJB, Tonneijck L, Smits MM, Emanuel AL, et al. The renal hemodynamic effects of the SGLT2 inhibitor dapagliflozin are caused by post-glomerular vasodilatation rather than pre-glomerular vasoconstriction in metformin-treated patients with type 2 diabetes in the randomized, double-blind RED trial. Kidney Int. 2020;97(1):202-12.

27 Schnermann J, Briggs JP. Tubuloglomerular feedback: mechanistic insights from genemanipulated mice. Kidney Int. 2008;74(4): 418-26.

28 Pessoa TD, Campos LC, Carraro-Lacroix L, Girardi AC, Malnic G. Functional role of glucose metabolism, osmotic stress, and sodiumglucose cotransporter isoform-mediated transport on $\mathrm{Na}+/ \mathrm{H}+$ exchanger isoform 3 activity in the renal proximal tubule. J Am Soc Nephrol. 2014;25(9):2028-39.

29 Onishi A, Fu Y, Patel R, Darshi M, CrespoMasip M, Huang W, et al. A role for tubular $\mathrm{Na}+\mathrm{H}+$ exchanger NHE3 in the natriuretic effect of the SGLT2 inhibitor empagliflozin. Am J Physiol Renal Physiol. 2020;319(4): F712-28.

30 Vallon V, Gerasimova M, Rose MA, Masuda T, Satriano J, Mayoux E, et al. SGLT2 inhibitor empagliflozin reduces renal growth and albuminuria in proportion to hyperglycemia and prevents glomerular hyperfiltration in diabetic Akita mice. Am J Physiol Renal Physiol. 2014:306(2):F194-204.

31 Miyata KN, Zhao S, Wu CH, Lo CS, Ghosh A, Chenier I, et al. Comparison of the effects of insulin and SGLT2 inhibitor on the renal renin-angiotensin system in type 1 diabetes mice. Diabetes Res Clin Pract. 2020;162: 108107.

32 Gallo LA, Ward MS, Fotheringham AK, Zhuang A, Borg DJ, Flemming NB, et al. Once daily administration of the SGLT2 inhibitor, empagliflozin, attenuates markers of renal fibrosis without improving albuminuria in diabetic db/db mice. Sci Rep. 2016;6:26428.
33 Kidokoro K, Cherney DZI, Bozovic A, Nagasu H, Satoh M, Kanda E, et al. Evaluation of glomerular hemodynamic function by empagliflozin in diabetic mice using in vivo imaging. Circulation. 2019;140(4):303-15.

34 Mazidi M, Rezaie P, Gao HK, Kengne AP. Effect of sodium-glucose cotransport-2 inhibitors on blood pressure in people with type 2 diabetes mellitus: a systematic review and meta-analysis of 43 randomized control trials with 22,528 patients. J Am Heart Assoc. 2017; 6(6): 004007

35 Rosenstock J, Marquard J, Laffel LM, Neubacher D, Kaspers S, Cherney DZ, et al. Empagliflozin as adjunctive to insulin therapy in type 1 diabetes: the EASE trials. Diabetes Care. 2018;41(12):2560-9.

36 McCrimmon RJ, Henry RR. SGLT inhibitor adjunct therapy in type 1 diabetes. Diabetologia. 2018;61(10):2126-33.

37 Woods TC, Satou R, Miyata K, Katsurada A, Dugas CM, Klingenberg NC, et al. Canagliflozin prevents intrarenal angiotensinogen augmentation and mitigates kidney injury and hypertension in mouse model of type 2 diabetes mellitus. Am J Nephrol. 2019;49(4): $331-42$.

38 Kojima N, Williams JM, Takahashi T, Miyata N, Roman RJ. Effects of a new SGLT2 inhibitor, luseogliflozin, on diabetic nephropathy in T2DN rats. J Pharmacol Exp Ther. 2013; 345(3):464-72.

39 Lambers Heerspink HJ, de Zeeuw D, Wie L, Leslie B, List J. Dapagliflozin a glucose-regulating drug with diuretic properties in subjects with type 2 diabetes. Diabetes Obes Metab. 2013;15(9):853-62.

40 Heerspink HJ, Perkins BA, Fitchett DH, Husain M, Cherney DZ. Sodium glucose cotransporter 2 inhibitors in the treatment of diabetes mellitus: cardiovascular and kidney effects, potential mechanisms, and clinical applications. Circulation. 2016;134(10):752-72.

41 Cherney DZ, Perkins BA, Soleymanlou N, Xiao F, Zimpelmann J, Woerle HJ, et al. Sodium glucose cotransport-2 inhibition and intrarenal RAS activity in people with type 1 diabetes. Kidney Int. 2014;86(5):1057-8.

42 Yoshimoto T, Furuki T, Kobori H, Miyakawa M, Imachi H, Murao K, et al. Effects of sodium-glucose cotransporter 2 inhibitors on urinary excretion of intact and total angiotensinogen in patients with type 2 diabetes. J Investig Med. 2017;65(7):1057-61.

43 Lo CS, Miyata KN, Zhao S, Ghosh A, Chang SY, Chenier I, et al. Tubular deficiency of heterogeneous nuclear ribonucleoprotein $\mathrm{F}$ elevates systolic blood pressure and induces glycosuria in mice. Sci Rep. 2019;9(1):15765.

44 Alshnbari AS, Millar SA, O’Sullivan SE, Idris I. Effect of sodium-glucose cotransporter-2 inhibitors on endothelial function: a systematic review of preclinical studies. Diabetes Ther. 2020;11(9): 1947-63.
45 Han JH, Oh TJ, Lee G, Maeng HJ, Lee DH, Kim KM, et al. The beneficial effects of empagliflozin, an SGLT2 inhibitor, on atherosclerosis in ApoE-/- mice fed a Western diet. Diabetologia. 2017;60(2):364-76.

46 Mancini SJ, Boyd D, Katwan OJ, Strembitska A, Almabrouk TA, Kennedy S, et al. Canagliflozin inhibits interleukin-1 $\beta$-stimulated cytokine and chemokine secretion in vascular endothelial cells by AMP-activated protein kinase-dependent and -independent mechanisms. Sci Rep. 2018;8(1):5276.

47 Gaspari T, Spizzo I, Liu H, Hu Y, Simpson RW, Widdop RE, et al. Dapagliflozin attenuates human vascular endothelial cell activation and induces vasorelaxation: a potential mechanism for inhibition of atherogenesis. Diab Vasc Dis Res. 2018;15(1):64-73.

48 Uthman L, Homayr A, Juni RP, Spin EL, Kerindongo R, Boomsma $\mathrm{M}$, et al. Empagliflozin and dapagliflozin reduce ROS generation and restore NO bioavailability in tumor necrosis factor $\alpha$-stimulated human coronary arterial endothelial cells. Cell Physiol Biochem. 2019; 53(5):865-86.

49 El-Daly M, Pulakazhi Venu VK, Saifeddine M, Mihara K, Kang S, Fedak PWM, et al. Hyperglycaemic impairment of PAR2-mediated vasodilation: prevention by inhibition of aortic endothelial sodium-glucose-co-transporter-2 and minimizing oxidative stress. Vascul Pharmacol. 2018;109:56-71.

50 Khemais-Benkhiat S, Belcastro E, IdrisKhodja N, Park SH, Amoura L, Abbas M, et al. Angiotensin II-induced redox-sensitive SGLT1 and 2 expression promotes high glucose-induced endothelial cell senescence. J Cell Mol Med. 2020;24(3):2109-22.

51 Maki T, Maeno S, Maeda Y, Yamato M, Sonoda N, Ogawa Y, et al. Amelioration of diabetic nephropathy by SGLT2 inhibitors independent of its glucose-lowering effect: a possible role of SGLT2 in mesangial cells. Sci Rep. 2019;9(1):4703.

52 Liu Y. Cellular and molecular mechanisms of renal fibrosis. Nat Rev Nephrol. 2011;7(12): 684-96.

53 Li J, Liu H, Takagi S, Nitta K, Kitada M, Srivastava $\mathrm{S}$. P, et al. Renal protective effects of empagliflozin via inhibition of EMT and aberrant glycolysis in proximal tubules. JCI Insight. 2020;5(6):e129034.

54 Heerspink HJL, Perco P, Mulder S, Leierer J, Hansen MK, Heinzel A, et al. Canagliflozin reduces inflammation and fibrosis biomarkers: a potential mechanism of action for beneficial effects of SGLT2 inhibitors in diabetic kidney disease. Diabetologia. 2019;62(7): 1154-66.

55 Panchapakesan U, Pegg K, Gross S, Komala MG, Mudaliar H, Forbes J, et al. Effects of SGLT2 inhibition in human kidney proximal tubular cells-renoprotection in diabetic nephropathy? PLoS One. 2013;8(2):e54442. 
56 Huang F, Zhao Y, Wang Q, Hillebrands JL, van den Born J, Ji L, et al. Dapagliflozin attenuates renal tubulointerstitial fibrosis associated with type 1 diabetes by regulating STAT1/TGF $\beta 1$ signaling. Front Endocrinol. 2019;10:441.

57 Zhao Y, Xu L, Tian D, Xia P, Zheng H, Wang $\mathrm{L}$, et al. Effects of sodium-glucose co-transporter 2 (SGLT2) inhibitors on serum uric acid level: a meta-analysis of randomized controlled trials. Diabetes Obes Metab. 2018; 20(2):458-62.
58 Novikov A, Fu Y, Huang W, Freeman B, Patel $\mathrm{R}$, van Ginkel C, et al. SGLT2 inhibition and renal urate excretion: role of luminal glucose, GLUT9, and URAT1. Am J Physiol Renal Physiol. 2019;316(1):F173-85.

59 Herrington WG, Preiss D, Haynes R, von Eynatten M, Staplin N, Hauske SJ, et al. The potential for improving cardio-renal outcomes by sodium-glucose co-transporter- 2 inhibition in people with chronic kidney disease: a rationale for the EMPA-KIDNEY study. Clin Kidney J. 2018;11(6):749-61.

60 Dekkers CCJ, Gansevoort RT. Sodium-glucose cotransporter 2 inhibitors: extending the indication to non-diabetic kidney disease? Nephrol Dial Transplant. 2020;35(Suppl 1): i33-42.
61 Fernandez-Fernandez B, Sarafidis P, Kanbay M, Navarro-González JF, Soler MJ, Górriz JL, et al. SGLT2 inhibitors for non-diabetic kidney disease: drugs to treat CKD that also improve glycaemia. Clin Kidney J. 2020;13(5): 728-33.

62 Li D, Wang T, Shen S, Fang Z, Dong Y, Tang $\mathrm{H}$. Urinary tract and genital infections in patients with type 2 diabetes treated with sodium-glucose co-transporter 2 inhibitors: a meta-analysis of randomized controlled trials. Diabetes Obes Metab. 2017;19(3):348-55. 